\title{
BMJ Global Health Refocusing vitamin A supplementation programmes to reach the most vulnerable
}

\author{
Erin McLean (D) , ${ }^{1}$ Rolf Klemm, ${ }^{2,3}$ Hamsa Subramaniam, ${ }^{4}$ Alison Greig ${ }^{5}$
}

To cite: McLean E, Klemm R, Subramaniam $\mathrm{H}$, et al. Refocusing vitamin $\mathrm{A}$ supplementation programmes to reach the most vulnerable. BMJ Global Health 2020;5:e001997. doi:10.1136/ bmjgh-2019-001997

Handling editor Seye Abimbola

Received 15 September 2019 Revised 30 April 2020 Accepted 1 May 2020
Check for updates

(C) Author(s) (or their employer(s)) 2020. Re-use permitted under CC BY-NC. No commercial re-use. See rights and permissions. Published by BMJ.

${ }^{1}$ Nutrition, UNICEF, New York City, New York, USA

${ }^{2}$ Helen Keller International, New York City, New York, USA

${ }^{3}$ Johns Hopkins University, Baltimore, Maryland, USA ${ }^{4}$ UNICEF, New York City, New York, USA

${ }^{5}$ Nutrition International, Ottawa, Ontario, Canada

Correspondence to

Dr Erin McLean;

edmclean_75@hotmail.com

\section{ABSTRACT}

WHO recommends vitamin A supplementation (VAS) programmes for children 6-59 months where vitamin A deficiency is a public health problem. However, resources for VAS are falling short of current needs and programme coverage is suffering. The authors present the case for considering the options for shifting efforts and resources from a generalised approach, to prioritising resources to reach populations with continued high child mortality rates and high vitamin A deficiency prevalence to maximise child survival benefits. This includes evaluating where child mortality and/or vitamin A deficiency has dropped, as well as using under 5 mortality rates as a proxy for vitamin A deficiency, in the absence of recent data. The analysis supports that fewer countries may now need to prioritise VAS than in the year 2000, but that there are still a large number of countries that do. The authors also outline next steps for analysing options for improved targeting and cost-effectiveness of programmes. Focusing VAS resources to reach the most vulnerable is an efficient use of resources and will continue to promote young child survival.

\section{PRIORITISATION OF NATIONAL VITAMIN A SUPPLEMENTATION PROGRAMMES IN THE CONTEXT OF DROPPING COVERAGE}

WHO recommends initiating vitamin $\mathrm{A}$ supplementation (VAS) programmes for children 6-59 months of age in settings where vitamin A deficiency (VAD) is a public health problem, to reduce child morbidity and mortality. ${ }^{1}$ Simultaneously, countries are expected to develop and implement interventions that sustainably increase availability and consumption of nutrient-rich foods and improve overall public health conditions to eliminate VAD, so that VAS will no longer be required. This is because efforts to ensure the population can meet their dietary requirements through available and affordable nutritious food obviates the need for supplementation, which is a short-term intervention to mitigate the impact of VAD on mortality. ${ }^{2}$ While the relationship between VAD and mortality is not linear, evidence demonstrates

\section{Summary box}

- Coverage of vitamin A supplementation programmes, a key intervention for child survival, has slipped dramatically, starting in 2016, especially in regions where child morbidity and mortality remain very high.

- The authors assessed the continued need for programmes through analysing recent data on vitamin A deficiency and under 5 child mortality, including from infectious causes, on which vitamin A supplementation programmes may have an impact.

- The analysis showed that while a small number of countries no longer need vitamin A supplementation programmes, due to their success in reducing vitamin A deficiency and under 5 mortality, the need remains high in many countries, especially given the slow progress towards improving dietary intake of vitamin $\mathrm{A}$.

- However, lack of recent data limited the ability to adequately analyse the situation in some countries. There is a strong need for improved availability of nationally representative data.

- Because the availability of data will not be immediately resolved, the authors propose additional analyses that may offer evidence to refine target population selection, including to a younger age group.

an association between high VAD prevalence and high child mortality" ${ }^{4}$ Therefore, once vitamin A nutrition through the diet is adequate, VAS programmes can be scaled back.

The process of scaling back requires data from a number of sources to guide VAS programme decisions. These include data from: dietary intake surveys; programme coverage data of interventions that increase vitamin A intake in children, including fortification and micronutrient powders; periodic nationally representative micronutrient status surveys that include appropriate vitamin A biomarkers and consideration of overall child mortality rates. That said, few countries have reliable and current data on all of these 
domains. The Global Alliance for Vitamin A (GAVA) recommends scaling back programmes when there is evidence that vitamin A intake is sufficient and VAD is present in $<10 \%$ of children $6-59$ months. ${ }^{5}$ This lower cut-off is to avoid dismantling a public health programme in settings where VAD remains elevated and dietary intake remains low. Until a reduction in VAD is achieved, VAS programmes remain a critical child survival intervention especially in countries with high levels of child mortality.

Global commitment for VAS programmes appears to be waning, particularly worrying is that this is also happening in contexts where the programme is critically needed. An analysis published in 2018 demonstrated that annual coverage with two doses of VAS dropped dramatically in 2016 and that the drop was concentrated in populations exhibiting high child mortality rates and therefore most likely to benefit from the intervention. ${ }^{6}$ The data for 2017 demonstrated that this trend continued and may be worsening. ${ }^{7}$ These analyses were possible because UNICEF maintains a global VAS coverage database, which tracks national programmes in 82 selected countries, identified as needing VAS programmes in the year 2000 .

Reasons for lower coverage rates include the shift in financing around supplementary immunisation activities, as global goals for polio eradication are within reach. This is particularly true in West and Central Africa, where polio financing has assured a delivery mechanism for VAS in a large number of countries. ${ }^{6}$ Campaign fatigue may also be a contributing factor as the global health community focuses on strengthening health systems to achieve sustained improvements in the quality and coverage of essential health and nutrition interventions. Due to the need to urgently improve child survival through high VAS coverage, VAS was delivered through special events or campaigns such as Child Health Days and/or piggybacked on National Immunisation Days which bypassed and perhaps disrupted usual health services. ${ }^{8}$

The drop in VAS coverage has come at the same time as questions have arisen on whether VAS is still needed, with some stakeholders citing a changing context with respect to infection, deaths, and VAD and vitamin A intake. ${ }^{9}{ }^{10}$ However, assessing these changes is not simple or straightforward. Even in countries that have recently completed national micronutrient surveys, many surveys report only retinol binding protein (RBP) and there have been concerns about the adequacy of RBP to accurately reflect VAD in a population, including low sensitivity. ${ }^{11} 12$ In the most recent global VAD estimates, which revealed little change between 1991 and 2013 in regional VAD prevalence in South Asia and sub-Saharan Africa, authors noted the lack of recent data as a challenge to generating estimates, ${ }^{10}$ suggesting the need for a proxy indicator for VAD until national vitamin A (VA) status data are available. In the absence of VA status data, we examine if high under 5 mortality rates (U5MR) may serve as a useful proxy.

In this paper, we present an analysis that examines whether levels of U5MR can be used as a proxy to justify continued universal VAS in countries, and use this as a basis for revising the list of countries for which VAS programmes and global VAS coverage tracking should be prioritised.

\section{CRITERIA TO ASSESS THE NEED FOR NATIONAL PREVENTIVE VAS PROGRAMMES}

U5MR is both an indication of the possible scale of impact for this intervention, given its impact on mortality reduction, and also can be a proxy for VAD in countries where data do not exist. ${ }^{13}$

As an indication of scale of impact, it is well known that at higher rates of U5MR, there are more deaths from infectious causes. ${ }^{14}$ So, in populations with a higher U5MR, the impact of VAS on mortality would be likely greater, if all other things are equal. Employing the same logic, in a population with a lower U5MR (ie, $<25$ per 1000 births), the potential for VAS to impact on mortality would be less. Because of this, there is value in identifying a threshold or "cut-off" to reconsider the significance of a VAS programme in that setting. The Sustainable Development Goal (SDG) on healthy lives has a target for reducing preventable deaths to a U5MR less than 25, in part because this is expected when infectious causes are greatly. ${ }^{1516}$ Analyses of U5MR over time have revealed that infectious causes have decreased not just in numbers, but also proportionally over time in all countries. ${ }^{14}$ The largest reductions in deaths between 2006 and 2016 were those from various infectious causes. Specifically, measles saw the largest reduction as a cause of death in both the postneonatal period and children $1-4$ years. ${ }^{14}$ We also reviewed the Global Burden of Disease data by region, looking at postneonatal and deaths in children 1-4 years in 1990, 2000, 2010 and 2017, and noted the reduction in proportion of infectious causes of deaths, but that the slope of the curve was flattest in Africa, followed by South Asia. ${ }^{14}$

The changing epidemiology of deaths is not limited to the shift in causes of death, but also to age groups, with a proportional increase in the number of younger children under 5 . Given that the age range of supplementation is children 6-59 months of age, the relative burden of neonatal deaths vs child deaths is an important profile to consider when assessing the potential impact VAS can have on mortality, since current VAS programmes begin at 6 months of age and would not address deaths in the neonatal period. Recent regional mortality estimates based on Global Burden of Disease regions indicate that neonatal deaths are far greater than child (1-4 years) deaths in some settings, such as in South Asia where there are 23.2 neonatal deaths per 1000 as compared with 7.4 child deaths per $1000 .{ }^{14}$ In sub-Saharan Africa as a whole, the numbers are more equal, with 25.9 neonatal deaths and 28.3 child deaths per 1000, although in West Africa, the relationship changes to 31.6 neonatal deaths as compared with 40.7 child deaths, revealing the importance of deaths in 
Table 1 Sensitivity and specificity for VAD threshold of $20 \%$ with identified U5MR thresholds

\begin{tabular}{lll}
\hline Cut-off & Sensitivity (CLD) & Specificity (CLD) \\
\hline 35 & $78.6(0.30)$ & $0.66(0.32)$ \\
40 & $\mathbf{8 1 . 1}(0.27)$ & $\mathbf{8 2 . 8}(0.30)$ \\
45 & $79.5(0.27)$ & $85.2(0.30)$ \\
50 & $77.5(0.28)$ & $85.7(0.31)$ \\
\hline
\end{tabular}

Sensitivity and specificity values approximating $80 \%$ or above were considered desirable to reliably identify countries with significant VAD, identified in bold italics.

Rows in bold italics show U5MR thresholds offering values optimizing both sensitivity and specificity, in this case approximating $>=80 \%$ for both.

CLD, Confidence limit differences; U5MR, under 5 mortality rates; $\mathrm{VAD}$, vitamin A deficiency.

older children that remains in this setting. ${ }^{14}$ These data highlight the importance of context specificity in decision making around VAS.

To identify settings most in need of VAS support, we conducted an analysis in two parts. First, we sought an appropriate U5MR measure to serve as a proxy for VAD in countries with no VAD data. To do this, we used nationally representative surveys from 1999 to 2018 measuring serum retinol and compared them to the estimated U5MR for the same year. Table 1 shows the sensitivity and specificity of population $\mathrm{VAD} \geq 20 \%$ at different cutoffs of U5MR, alongside the accompanying confidence limit differences (CLD). CLD is the difference between the upper and lower value of the confidence interval to demonstrate estimate precision - lower values indicate increasing precision of the estimate.

A U5MR of 40 provided reasonable sensitivity and specificity in determining the populations that had a VAD $\geq 20 \%$. Given the imperfection of the proxy, and the WHO recommendation for VAD of $20 \%$ to start a programme, we wanted to assess U5MR as a predictor of this level of VAD. Furthermore, with a U5MR at this level, populations are also still experiencing excess mortality from infectious causes.

The goal of the second part of our analysis was to use U5MR or survey data to identify countries where VAS should remain a priority intervention. We selected priority countries with VAD $>20 \%$ based on reliable nationally representative survey data collecting either serum retinol or RBP within the last 15 years, or with $\mathrm{U} 5 \mathrm{MR}>40$ if such data were not available. In the final application of the criteria and the review of countries, there was sometimes a need to take older data into account for final decision-making. This is further detailed in box 1 .

\section{HOW DID WE APPLY THE CRITERIA ON U5MR AND VAD TO ALL COUNTRIES?}

We applied the new criteria in four phases (box 1), first by using recent VAD prevalence data (or a proxy
Box 1 Application of considerations for the need for national vitamin A supplementation (VAS) programmes

\section{Phase 1}

Identify which countries qualify for inclusion on the list based on evidence of vitamin A deficiency (VAD), either as measured or by proxy of deficiency:

1. Evidence of VAD as a moderate public health problem (definition: a national VAD survey in the last 15 years with VAD $\geq 10 \%$ in children 6-59 months).

2. OR, in the absence of VAD data, a under five mortality rates (U5MR) $>40$ for inclusion.

\section{Phase 2}

Identify which countries do not qualify, by applying a lower U5MR limit (25), given the expected reduced impact of VAS in low U5MR settings.

\section{Phase 3}

Asses countries suggested as additions and determine the expected benefit of initiating a VAS programme.

\section{Phase 4}

Asses countries suggested for removal and determine the context and implications of stopping the programme immediately.

for it), second by imposing a lower bound U5MR limit, third by reviewing countries which met or did not meet the criteria, and finally determining if contextual country-specific factors may influence the results of this approach.

\section{WHAT WAS LEARNED IN USING THIS EVIDENCE-BASED APPROACH FOR NATIONAL VAS PROGRAMMES?}

Proxy indicators are needed in the interim when VAD data are not available. As previously mentioned, GAVA recommends scaling back programmes when there is evidence that vitamin A intake is sufficient and VAD is present in $<10 \%$ of children $6-59$ months. As previously documented $(8,15)$, the current review of country data confirmed that it is difficult for most countries to make decisions based on their vitamin A nutrition because they lack recent data on VA status-only 38 of the 89 which were ultimately assessed had VAD data available within the inclusion time frame, six of them conducted within the past 5 years-and vitamin A intake of risk groups. Of the 38 countries with VAD data, only 31 of these were on the initial list for global programme tracking. Further, most countries do not collect other indicators of vitamin A nutrition, such as breast milk retinol, modified relative dose response (MRDR) or biological indicators of excess intakes.

Our analysis also confirmed that there are countries which had been on the UNICEF global tracking list since 2000 , but no longer need a national VAS programme, either due to a reduction in VAD since that time, or because mortality has significantly dropped even though VAD is still present (four countries). It also revealed that some countries could be added since seven countries not on the list had elevated VAD, although we rejected 
adding these because they were considered low risk for VAD-related mortality.

For the countries that were assessed more closely after screening, fourteen countries were dropped due to reduced VAD and/or U5MR, while four countries were dropped due to very low U5MR $(<25)$, even in the presence of elevated VAD. The 12 countries that were retained consisted of five countries with U5MR close to 40 and historical VAD with no recent VAD data; four countries with VAD close to $10 \%$ and still relatively elevated U5MR, and three countries where there were remaining questions about data or surveys that were about to be finalised. This suggests that more countries could drop in the next few years.

Ultimately, a 'one size, fits all' approach is not possible. The described approach is a first step to identify countries where VAS should be prioritised. It could be that a single survey shows low VAD in a country, but a high U5MR and scant corroborating evidence (ie, increasing intake of vitamin A-rich foods) explaining the decline in $\mathrm{VAD}$, which muddies the overall picture. These data are conflicting, therefore, justifying a conservative approach and a continued VAS programme given the lack of corroborating evidence that VAS would no longer be beneficial. GAVA is working on establishing clearer guidance for countries in this situation. Of course, any recommendation for additional data collection (biomarker, dietary intake or other) in resource-limited environments needs to be fit-for-purpose and cost-effective.

Our analysis revealed that many countries on the original list now have a substantially lower U5MR than in the year 2000. This, alongside what we know about the changing profile of deaths, raises questions about the relative concentration of deaths in younger children and the potential need to refocus the age group for VAS programmes.
Overall, implementing the approach reduced the list of countries where national efforts are needed and should be tracked. We feel this is an important step in targeting limited resources to areas of greatest need. Specifically, it reduced the list of countries that the UNICEF global VAS coverage database tracks from 82 countries to 64 (figure 1). This also revealed that progress has indeed been made in some settings either on VAD reduction through increasing dietary intake, or on U5MR reduction, or on both since this list was generated from similar criteria for the year 2000 (VAD and/or elevated U5MR).

\section{HOW CAN LIMITED RESOURCES FOR VAS PROGRAMMES BE FURTHER FOCUSED FOR THE BIGGEST IMPACT?}

As this and previous analyses have demonstrated, data are often lacking to inform evidence-based programming. ${ }^{10}{ }^{17}$ High-quality, population-based vitamin A status surveys are needed in many high priority countries. This is important not just to identify populations with VAD to justify VAS programmes, but also to inform and accelerate strategies needed to sustainably increase vitamin A intake so that VAS programmes are no longer needed. This is critical for many countries.

We cannot let coverage remain low or drop further for the countries in need of VAS programmes, especially given the SDG focus on eliminating preventable child mortality. Given the global shift in strategies, there is a need to identify how best to deliver VAS to all children in need two times per year. Furthermore, documentation of new approaches is critical to share knowledge and inform other countries.

This paper focused on identifying where national VAS programmes are needed and are likely to have the biggest impact, given our current knowledge. However, there are additional analyses that need to be undertaken to ensure resources are optimised and that those who will benefit most from VAS programmes are reached. First, an analysis

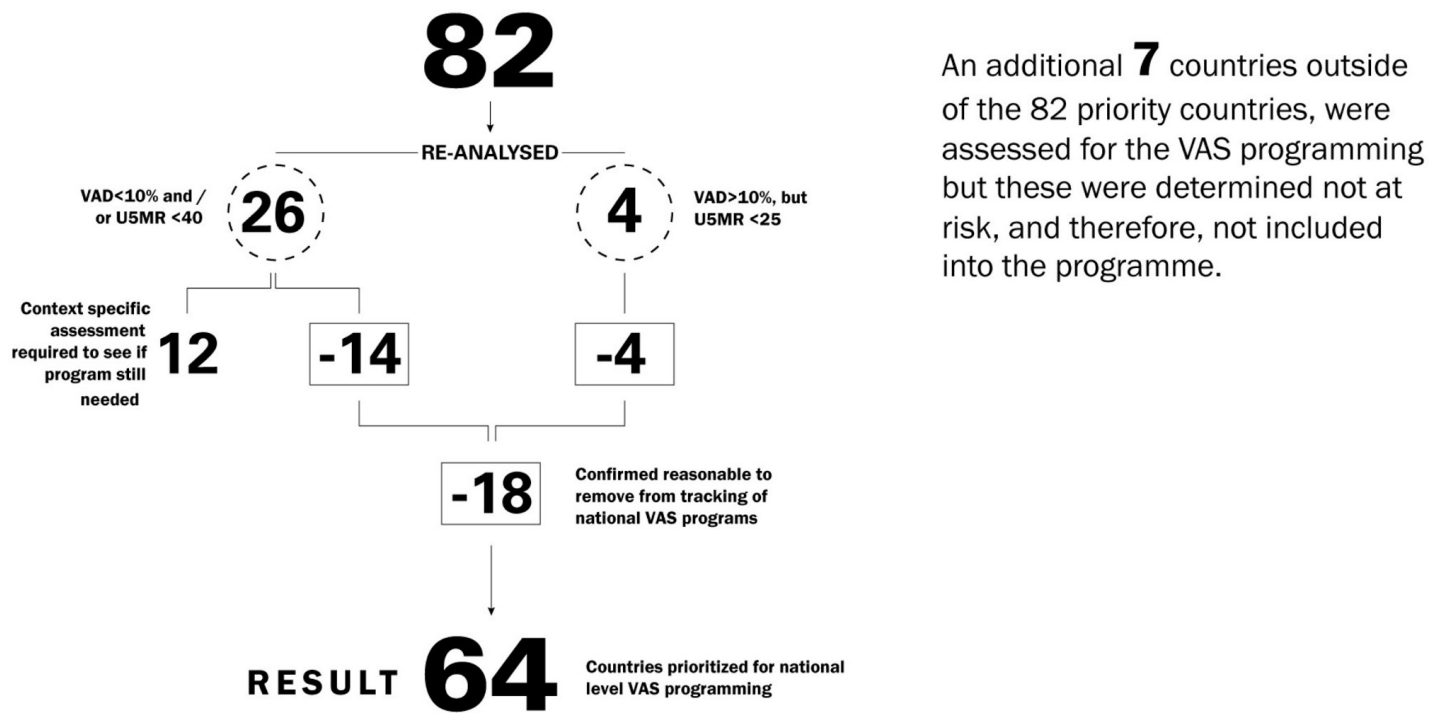

Figure 1 Review of countries for inclusion list for global tracking of national vas programmes. VAS, vitamin A supplementation; VAD, vitamin A deficiency. 
is needed to determine if some parts of the target population should be prioritised, due to vulnerability related to mortality rates or vitamin A intake. It is not possible to target based on clinical signs of xerophthalmia because these are now rare, but we know that there are serious health risks, including elevated mortality associated with subclinical VAD. With the changing global context, there may be value in focusing VAS to a narrow and younger age range than the current 6-59 months, as the mortality data suggest. ${ }^{14}$ Second, an assessment of the possible delivery approaches and their costs to reach children, including the most vulnerable, is needed to inform decisions. As was mentioned in the beginning of this paper, a shift in global financing and other factors have resulted in the loss of some delivery platforms. An assessment is needed to determine the most cost-effective ways to reach the target population to ensure efficient use of limited resources.

With the completion of this initial analysis, GAVA partners have committed to looking deeper at how best to support countries in prioritising limited resources in the years ahead. The additional analyses, including agespecific assessments of vitamin A intake and mortality, will require convening additional stakeholders and expertise. Reducing the list of countries prioritised for national VAS programmes is a first step to targeting limited resources and ensuring VAS coverage is maintained where it has the potential to achieve the greatest impact.

Contributors EM conceptualised the approach to revising the list of countries tracked for needing national programmes and worked on a first draft of this paper. RK provided input into the approach and drafts of the paper. HS conducted the analysis to use U5MR as a proxy indicator and provided input into the drafts of the paper. AG provided input into the approach and drafts of the paper.

Funding We are grateful to Global Affairs Canada and Proctor and Gamble for funding for vitamin A supplementation, which supported some staff and consultancy time to undertake this analysis and write this paper.

Competing interests The authors work for organisations that support vitamin A supplementation programmes.

Patient consent for publication Not required.

Provenance and peer review Not commissioned; externally peer reviewed.

Data availability statement Data are available upon request.

Open access This is an open access article distributed in accordance with the Creative Commons Attribution Non Commercial (CC BY-NC 4.0) license, which permits others to distribute, remix, adapt, build upon this work non-commercially, and license their derivative works on different terms, provided the original work is properly cited, appropriate credit is given, any changes made indicated, and the use is non-commercial. See: http://creativecommons.org/licenses/by-nc/4.0/.

\section{ORCID iD}

Erin McLean http://orcid.org/0000-0002-6273-593X

\section{REFERENCES}

1 World Health Organization. Guideline : Vitamin A supplementation in infants and children $6-59$ months of age. World Health Organization, 2011.

2 Palmer AC, West KP, Dalmiya N, et al. The use and interpretation of serum retinol distributions in evaluating the public health impact of vitamin A programmes. Public Health Nutr 2012;15:1201-15.

3 Sommer A, Tarwotjo I, Djunaedi E, et al. Impact of vitamin A supplementation on childhood mortality. A randomised controlled community trial. Lancet 1986;1:1169-73.

4 Beaton GH, Martorell R, Aronson KA, et al. Vitamin A supplementation and child morbidity and mortality in developing countries. Food Nutr Bull 1994;15:1-9.

5 GAVA. Conditions for scaling back universal preschool vitamin A supplementation: policy brief, 2019. Available: http://www.gava. org/content/user_files/2019/05/GAVA-Brief-Scaling-Back-VAS-ENMay-10.pdf

6 McLean E, White J, Krasevec J, et al. Coverage at crossroads: new directions for vitamin A supplementation programmes. UNICEF, 2018.

7 UNICEF. Monitoring the Situation of Children and Women - Vitamin A Supplementation Interactive Dashboard, 2019.

8 Chan M. Ten years in public health 2007-2017. World Health Organization, 2017.

9 Mason J, Greiner T, Shrimpton R, et al. Vitamin A policies need rethinking. Int J Epidemiol 2015;44:283-92.

10 Stevens GA, Bennett JE, Hennocq Q, et al. Trends and mortality effects of vitamin A deficiency in children in 138 low-income and middle-income countries between 1991 and 2013: a pooled analysis of population-based surveys. Lancet Glob Health 2015;3:e528-36.

11 Whitehead R, Perrine C, Flores-Ayala R, et al. Use of modified relative dose-response to examine sensitivity and specificity of retinol binding protein as an indicator of vitamin A deficiency among Nepali children 6-23 months of age. FASEB J 2016;30.

12 Whitehead R, Perrine C, Mebrahtu S, et al. Defining a vitamin A deficiency cut-off for retionol binding protein in Nepal children 6-23 mo of age. FASEB J 2015.

13 Schultink W. Use of under-five mortality rate as an indicator for vitamin A deficiency in a population. J Nutr 2002;132:2881S-3.

14 GBD 2016 Causes of Death Collaborators. Global, regional, and national age-sex specific mortality for 264 causes of death, 19802016: a systematic analysis for the global burden of disease study 2016. Lancet 2017;390:1151-210.

15 United Nations Development Program. Goal 3: Good health and well-being. In: Sustainable development goals, 2016.

16 Liu L, Oza S, Hogan D, et al. Global, regional, and national causes of child mortality in 2000-13, with projections to inform post-2015 priorities: an updated systematic analysis. Lancet 2015;385:430-40.

17 Wirth JP, Petry N, Tanumihardjo SA, et al. Vitamin A supplementation programs and country-level evidence of vitamin A deficiency. Nutrients 2017;9. doi:10.3390/nu9030190. [Epub ahead of print: 24 Feb 2017]. 